\title{
La radiologia interventistica nel trattamento delle stenosi venose centrali nei pazienti con FAV dialitiche
}

\author{
Andrea Lovaria, Antonio Nicolini, Daniele Meregalia
}

\author{
Servizio di Radiologia IRCCS Ospedale Maggiore di Milano \\ Servizio di Radiologia Vascolare Padiglione Zonda, Milano
}

\section{$\mathbf{L}$} e stenosi del-le vene centrali, localizzate soprattutto a livello delle vene succlavie e anonime, costituiscono un grave problema nei pazienti portatori di FAV dialitiche degli arti superiori. Esse sono presenti con una frequenza che, secondo alcuni autori, raggiunge il $40 \%$ dei casi $(1,2)$.

L'eziologia di queste lesioni è dovuta a diversi fattori, fra i quali ricordiamo in ordine di importanza:

- il traumatismo vasale provocato dai cateteri utilizzati per la dialisi, prima dell'allestimento delle FAV;

- l'elevato flusso sanguigno indotto dalle FAV, che favorisce l'insorgenza di alterazioni parietali (iperplasia fibromiointimale);

- le turbolenze del flusso stesso in particolari sedi, quali le valvole venose, i tratti curvilinei e le confluenze, che si riscontrano per l'appunto a livello degli assi venosi prossimali.

Va sottolineato come le stenosi possano essere presenti, pur essendo asintomatiche, anche prima dell'allestimento delle FAV, soprattutto nei pazienti portatori di cateteri venosi centrali. In condizioni normali infatti, anche stenosi di discreta entità possono consentire un drenaggio venoso sufficiente, senza alcun sintomo. Ne deriva che è di fondamentale importanza lo studio flebografico degli assi venosi prossimali in tutti i pazienti candidati all'allestimento di FAV dialitiche degli arti superiori (3). Una volta confezionata la FAV, il notevole aumento del flusso ematico può rendere le stenosi emodinamicamente significative, aggravandone nel contempo l'entità. Il quadro clinico che ne deriva è costituito da edema spesso imponente dell'arto superiore, con malfunzionamento della FAV e inevitabile evoluzione verso la trombosi completa dell'asse venoso prossimale, con conseguente perdita dell'accesso vascolare dialitico. A volte l'evoluzione del quadro è molto rapida, più spesso lentamente progressiva, ma in ogni caso è indispensabile l'accertamento diagnostico mediante flebografia, eseguita con iniezione di mezzo di contrasto nella FAV dialitica. L'ecocolordoppler è infatti ostacolato dallo "sbarramento" costituito dalle strutture ossee dello stretto toracico superiore. Il trattamento di queste lesioni si esegue attualmente mediante le tecniche di radiologia interventistica, che ovviano alle difficoltà e ai rischi della chirurgia in questo particolare distretto venoso.

L'angioplastica percutanea transluminale (PTA) è stata largamente impiegata, con elevate percentuali di successo tecnico, ma con importanti limitazioni, dovute alla notevole resistenza di queste lesioni e all' "elastic recoil" della parete venosa, che provocano generalmente una rapida recidiva della stenosi (2). Per mantenere nel tempo la dilatazione indotta dalla PTA, vengono attualmente impiegate le endoprotesi metalliche o stents, già utilizzati con buoni risultati in altri distretti vascolari, a partire dai primi anni '80. L'impiego degli stents ha migliorato notevolmente le percentuali di pervietà a distanza, pur presentando il problema della ristenosi, dovuto all'iperplasia fibromiointimale, che costituisce la normale reazione all'impianto delle endoprotesi, e che è particolarmente "aggressiva" in questo distretto, nonostante il grosso diametro delle endoprotesi utilizzate. È pertanto indispensabile seguire accuratamente i pazienti, eseguendo un controllo flebografico in caso di recidiva dei sintomi che devono far so- 
spettare una ristenosi a livello dello stent. In tali casi, può essere agevolmente eseguita una nuova procedura di dilatazione, che solitamente consente il ripristino della pervietà del segmento venoso trattato (4-6).

Per quanto riguarda la tecnica di esecuzione, essa è schematizzabile nelle seguenti tappe fondamentali:

- flebografia preliminare per puntura della FAV;

- scelta della via di accesso, che, in caso di notevole tortuosità delle vene prossimali alla FAV, è generalmente più agevole mediante cateterismo retrogrado transfemorale;

- superamento della stenosi con una guida angiografica: in caso di stenosi serrate e irregolari, sono particolarmente utili le guide idrofiliche (Terumo Corporation);

- posizionamento di una guida rigida (tipo Amplatz extra-stiff), in grado di veicolare il catetere da angioplastica e quello contenente l'endoprotesi;

- dilatazione con un catetere a palloncino di diametro adeguato a quello delle vene (generalmente $10-12 \mathrm{~mm}$ ) in grado di sostenere elevate pressioni di gonfiaggio (oltre 15 atmosfere), per vincere la notevole resistenza delle stenosi, evitando nel contempo la rottura del palloncino. La dilatazione va mantenuta a lungo, fino alla completa scomparsa dell'impronta della stenosi sul pallone;

- controllo flebografico del risultato, con accurato reperaggio della stenosi residua, onde consentire un preciso posizionamento dell'endoprotesi;

- posizionamento di un'endoprotesi di diametro e lunghezza adatti al segmento venoso da trattare;

- controllo flebografico del corretto posizionamento ed eventuale dilatazione con lo stesso catetere da angioplastica, per favorire la completa espansione dello stent e la sua adesione alle pareti venose.

Per quanto riguarda gli stents utilizzati, essi devono avere particolari caratteristiche che li rendono adatti all'impiego in questo particolare distretto:

- elevata flessibilità per adattarsi alle curvature, spesso assai accentuate dell' asse venoso;

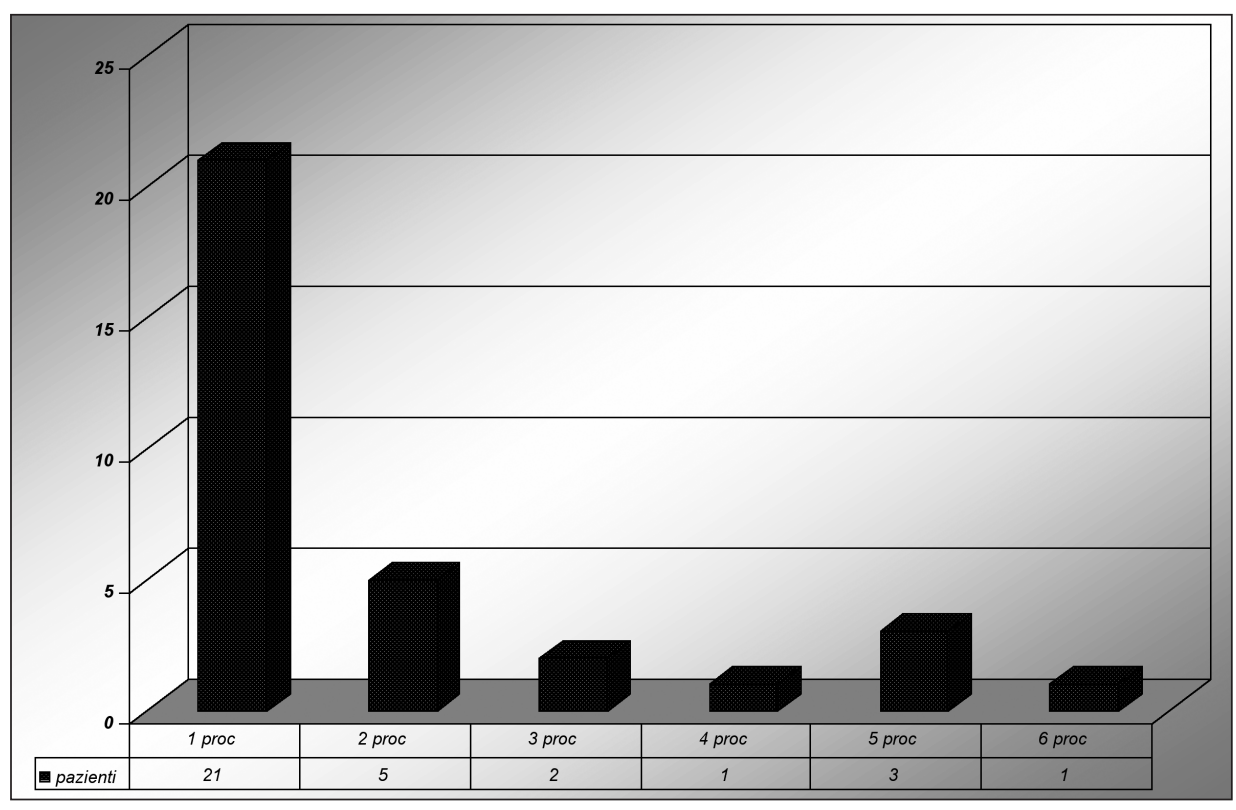

Fig. 1

- notevole forza di espansione radiale, per resistere al "recoil" della parete venosa;

- buona radiopacità, per poter posizionare agevolmente e con buona precisione l'endoprotesi;

- disponibilità in diametri e lunghezze adatti al segmento venoso da trattare.

Nella nostra esperienza, sono risultati particolarmente adatti i Wallstent (Boston Scientific) (attualmente disponibili nella versione "Easy", più facile da posizionare) e i Memotherm (Bard Angiomed). Entrambi sono del tipo autoespandibile, ma presentano caratteristiche leggermente diverse: i primi sono molto flessibili, adattandosi particolarmente ai segmenti venosi ricurvi, ma sono un po' più difficli da posizionare, a causa dell'accorciamento, anche se ridotto nell'ultima versione; i secondi consentono un posizionamento più preciso, ma sono nettamente più rigidi, trovando un impiego limitato a livello dei segmenti venosi più rettilinei, come le vene anonime (7).

\section{Esperienza personale}

La nostra esperienza si basa su una serie di 33 pazienti (18 maschi e 15 femmine, età media $=51.5$ anni, ran- ge $=5$-86). Tutti erano portatori di FAV dialitiche con sintomi (edema arto e/o malfunzionamento FAV) dovuti alla presenza di stenosi dell'asse venoso prossimale: in 22 pazienti erano presenti stenosi della vena succlavia, in 6 della vena anonima, 2 dell'iliaca esterna e in 1 della cava superiore. Dei 33 pazienti 9 sono stati trattati con PTA e 24 con PTA + stenting. Sono stati impiegati in totale 29 stents, del seguente tipo: 23 Wallstent, $4 \mathrm{Me}-$ motherm, 1 Perflex ed 1 AVE. Al follow-up (medio 20 mesi) sono stati valutati 19 pazienti, con il riscontro di 11 stenosi, 2 obliterazioni mentre 6 risultavano pervi.

Abbiamo dovuto riintervenire in 29 casi, eseguendo solo la PTA in 23, posizionando uno stent dopo PTA in 4 e un secondo stent in 2 . Sono state eseguite in totale 62 procedure nei 33 pazienti del nostro studio ed esattamente 1 in 21,2 in 5,3 in 2, 4 in 1,5 in 3 e 6 in 1 (Fig. 1).

Si sono avute 5 complicanze $(26.3 \%)$ dovute a scivolamento dello stent.

radzonda@polic.cilea.it 


\section{BIBLIOGRAFIA}

1. Schwab S, Quarles D, Middleton J, et al. Hemodialysis - associated subclavian vein stenosis. Kidney Int 1988; 33: 1156-9.

2. Glanz S, Gordon D, Lipkowitz et al. Axillary and subclavian vein stenosis: percutaneous angioplasty. Radiology 1988; 168: 371-3.

3. Surrat R, Picus D, Hicks M, et al. The importance of preoperative evaluation of the subclavian vein in dialysis access planning. AJR 1991; 156: 623-5.

4. Vesely TM, Hovsepian DM, Pilgram TK, et al. Upper extremity central venous obstruction in hemodialysis patients: treatment with Wallstents. Radiology 1997; 204: 343-8.

5. Eathard G. Percutaneous transvenous angioplasty in the treatment of vascular access stenosis. Kidney Int 1992; 42: 1390-7.

6. Schoenfeld R, Hermans H, Novick $A$, et al. Stenting of proximal venous obstructions to maintain hemodialysis. J Vasc Surg 1994; 19: 532-9.

7. Flueckiger F, Sternthal H, Klein G, et al. Strength, elasticity, and plasticity of expandable metal stents: in vitro studies with three types of stress. JVIR 1994; 5: 745 50 . 